\title{
Insight into Crystal Phase Dependent CO Dissociation on Rh Catalyst from DFT and Microkinetic Modeling
}

\author{
Xiaobin Hao a , Riguang Zhang ${ }^{\mathrm{a},}$, Lixia Ling ${ }^{\mathrm{a}}$, Maohong Fan ${ }^{\mathrm{b}, \mathrm{c}}$, Debao Li ${ }^{\mathrm{d}}$, Baojun Wang ${ }^{\mathrm{a},{ }^{*}}$ \\ a Key Laboratory of Coal Science and Technology of Ministry of Education and Shanxi Province, Taiyuan \\ University of Technology, Taiyuan 030024, Shanxi, P.R. China \\ b Department of Chemical Engineering and Department of Petroleum Engineering, University of Wyoming, \\ Laramie, WY 82071, USA \\ c School of Civil and Environmental Engineering, Georgia Institute of Technology, Atlanta, GA 30332 USA \\ d State Key Laboratory of Coal Conversion, Institute of Coal Chemistry, Chinese Academy of Sciences, Taiyuan \\ 030001, Shanxi, P.R. China
}

\section{Corresponding Author}

* zhangriguang@tyut.edu.cn (R. Z.); wangbaojun@tyut.edu.cn (B. W) 


\section{Microkinetic modeling}

In our study, the microkinetic modeling is used to examine the catalytic activity in the operation conditions considering the temperature, pressure, and the coverage of intermediates. The equilibrium constants for the step (1-1), (2-1) and (2-2) are calculated according to the following equation:1,2

$$
K=\exp \left[-\left(E_{\text {ads }}-T \Delta S\right) / R T\right]
$$

Where $E_{\text {ads }}$ is the adsorption energy of $\mathrm{CO}$ or $\mathrm{H}_{2}$ on the surface, while $\Delta S$ is the entropy change of gas-phase $\mathrm{CO}$ or $\mathrm{H}_{2}$, which are obtained from NIST Chemistry WebBook, ${ }^{3} R$ is molar gas constant $\left(8.314 \mathrm{~J} \cdot \mathrm{mol}^{-1} \cdot \mathrm{K}^{-1}\right)$. The other steps are described by the forward rate. And the rate constant $k_{\mathrm{n}}\left(k_{2}, k_{3}\right.$ and $k_{4}$ ) can be described as follows: ${ }^{4}$

$$
k_{\mathrm{n}}=\frac{k_{\mathrm{B}} T}{h} \frac{Q_{\mathrm{TS}}}{Q_{R}} \exp \left[-E_{a} / R T\right]
$$

Where $E_{\mathrm{a}}$ is the dissociation barrier for step (n), $k_{B}$ is Boltzmann constant $\left(1.38 \times 10^{-23} \mathrm{~J} \cdot \mathrm{K}^{-1}\right), h$ is Planck constant $\left(6.62 \times 10^{-34} \mathrm{~J} \cdot \mathrm{s}\right), Q_{\mathrm{TS}}$ and $Q_{\mathrm{R}}$ are the partition functions per unit volume for the transition state and reactant, respectively, which can be given by the following equation:

$$
Q=\frac{1}{\prod_{i=1}^{v i b}\left[1-\exp \left(-h v_{i} / k_{\mathrm{B}} T\right)\right]}
$$

Where $v_{i}$ is the vibration frequency. In our study, $T$ is equal to $543 \mathrm{~K}, P_{\mathrm{CO}}$ and $P_{\mathrm{H} 2}$ are equal to 4 and 8 atm. The equilibrium constants $K$ and rate constants $k_{n}$ at $543 \mathrm{~K}$ are presented in Table $\mathrm{S} 3$. For CO direct dissociation, as shown in Section 2.3, the step (1-2) is irreversible. Thus, CO direct dissociation rate can be given by

$$
r_{d i s}=k_{3} \theta_{\mathrm{CO}} \theta_{*}
$$

Where $\theta_{C O}$ and $\theta_{*}$ stand for the coverages of the adsorbed $\mathrm{CO}$ and free sites on $\mathrm{Rh}$ surface, respectively. Assuming $\mathrm{CO}$ adsorption and desorption are in equilibrium, $\theta_{C O}$ can be given by ${ }^{5}$

$$
\theta_{\mathrm{CO}}=P_{\mathrm{CO}} K_{1} \theta_{*}
$$


And the sum for the coverages of adsorbed $\mathrm{CO}$ and free active sites is equal to $1: \theta_{C O}+\theta_{*}=1$

Thus we can obtain that

$$
\theta_{*}=\frac{1}{P_{\mathrm{CO}} K_{1}+1}
$$

Thus, $\mathrm{CO}$ dissociation rate can be given by the following equation:

$$
r_{\mathrm{dis}}=k_{3} \frac{P_{\mathrm{CO}} K_{1}}{\left(P_{\mathrm{CO}} K_{1}+1\right)^{2}}
$$

For H-assisted dissociation, according to the reference, ${ }^{6}$ the dissociation rate can be given by the following equation.

$$
r_{\mathrm{dis}}=\frac{d \theta_{\mathrm{CH}}}{d_{\mathrm{t}}}=k_{4} \theta_{\mathrm{CHO}} \theta_{*} \text { or } r_{\mathrm{dis}}=\frac{d \theta_{\mathrm{C}}}{d_{\mathrm{t}}}=k_{4} \theta_{\mathrm{COH}} \theta_{*}
$$

Further, as shown in Table $\mathrm{S} 3$, the consumption reaction rate constant $\left(k_{4}\right.$ or $\left.\mathrm{k}_{-3}\right)$ of the intermediate $\mathrm{CHO}$ or $\mathrm{COH}$ is much larger than the generation reaction rate constant $\left(k_{3}\right)$. Thus, once the intermediate $\mathrm{CHO}$ or $\mathrm{COH}$ is generated, they will disappear immediately. Therefore, the instantaneous coverage of $\mathrm{CHO}$ or $\mathrm{COH}$ intermediates can be described as zero according to the steady-state approximation: ${ }^{7}$

$$
\frac{d \theta_{\mathrm{CHO}}}{d \mathrm{t}}=k_{3} \theta_{\mathrm{CO}} \theta_{\mathrm{H}}-k_{4} \theta_{\mathrm{CHO}} \theta_{*}-k_{-3} \theta_{\mathrm{CHO}} \theta_{*}=0 \text { or } \frac{d \theta_{\mathrm{COH}}}{d t}=k_{3} \theta_{\mathrm{CO}} \theta_{\mathrm{H}}-k_{4} \theta_{\mathrm{COH}} \theta_{*}-k_{-3} \theta_{\mathrm{COH}} \theta_{*}=0
$$

Thus, $\mathrm{H}$-assisted $\mathrm{CO}$ dissociation rate is given by the following equation:

$$
r_{\mathrm{dis}}=\frac{k_{3} k_{4}}{k_{4}+k_{-3}} \theta_{\mathrm{CO}} \theta_{\mathrm{H}}
$$

As shown in the above equation, the rate-determining step of $\mathrm{H}$-assisted $\mathrm{CO}$ dissociation on different $\mathrm{Rh}$ surfaces is determined by the specific conditions. When $k_{-3}$ is much smaller than $k_{4}, r_{\text {dis }}$ is largely determined by $k_{3} \theta_{\mathrm{CO}} \theta_{\mathrm{H}}$, the step (9) is the rate-determining step. However, when $k_{4}$ is much smaller than $k_{-3}$, the elementary step (10) is the rate-determining step; moreover, when $k_{3}$ is much smaller than $k_{-3}$, the elementary step (9) can be assumed to reach the equilibrium. Here, $r_{\text {dis }}$ can 
be equal to $k_{3} k_{4} \theta_{\mathrm{CO}} \theta_{\mathrm{H}} / k_{-3}$. $\theta_{\mathrm{CO}}$ is above-mentioned known and $\theta_{\mathrm{H}}$ is the coverage of the adsorbed $\mathrm{H}$ on $\mathrm{Rh}$ surface. Similar to $\theta_{\mathrm{CO}}, \theta_{\mathrm{H}}$ can be given by the following equation. ${ }^{5}$

$$
\theta_{\mathrm{H}}=P_{\mathrm{H}_{2}}^{1 / 2} K_{2}^{1 / 2} \theta_{*}
$$

At the same time, the sum for the coverages of the adsorbed species $(\mathrm{CO}, \mathrm{H}$ and $\mathrm{CHO}$ or $\mathrm{COH})$ and free active sites is equal to 1: $\theta_{\mathrm{CO}}+\theta_{\mathrm{H}}+\theta_{\mathrm{CHO}}+\theta_{*}=1$ or $\theta_{\mathrm{CO}}+\theta_{\mathrm{H}}+\theta_{\mathrm{COH}}+\theta_{*}=1$

Thus, we can obtain that

$$
\theta_{*}=\frac{1}{P_{\mathrm{co}} K_{1}+P_{\mathrm{H}_{2}}^{1 / 2} K_{2}^{1 / 2}+\frac{k_{3}}{k_{4}+k_{-3}} P_{\mathrm{co}} K_{1} P_{\mathrm{H}_{2}}^{1 / 2} K_{2}^{1 / 2}+1}
$$

Finally, we can obtain the equation for $\mathrm{H}$-assisted dissociation rate

$$
r_{\mathrm{dis}}=\frac{k_{3} k_{4}}{k_{-3}+k_{4}} \frac{P_{\mathrm{co}} K_{1} \cdot P_{\mathrm{H}_{2}}^{1 / 2} K_{2}^{1 / 2}}{\left(P_{\mathrm{co}} K_{1}+P_{\mathrm{H}_{2}}^{1 / 2} K_{2}^{1 / 2}+\frac{k_{3}}{k_{4}+k_{-3}} P_{\mathrm{co}} K_{1} P_{\mathrm{H}_{2}}^{1 / 2} K_{2}^{1 / 2}+1\right)^{2}}
$$




\section{The detailed description of surface models and adsorption sites}

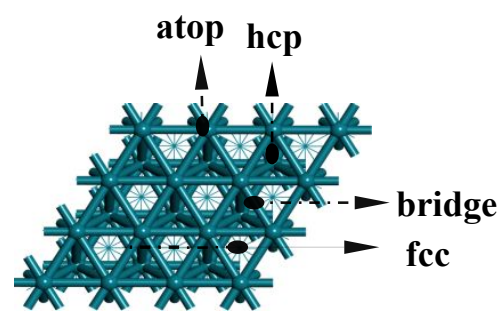

(a) (111)

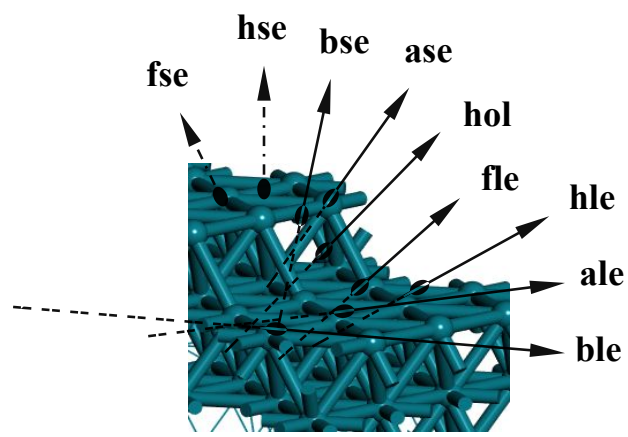

(d) (211)

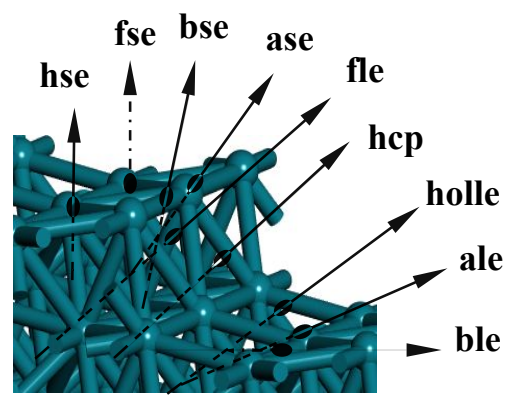

(g) (10-12)

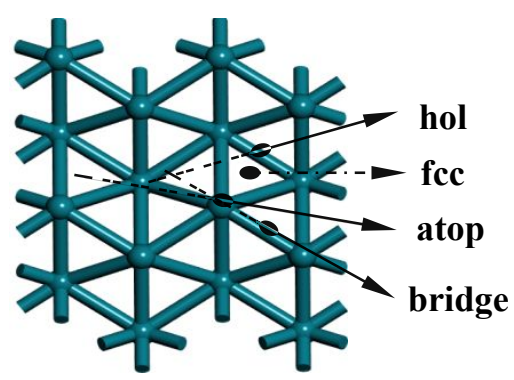

(J) (11-20)

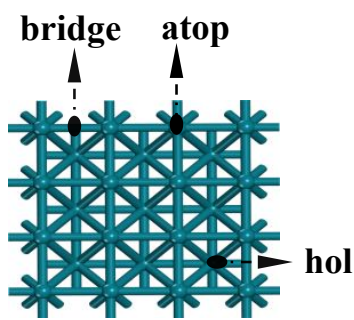

(b) (100)

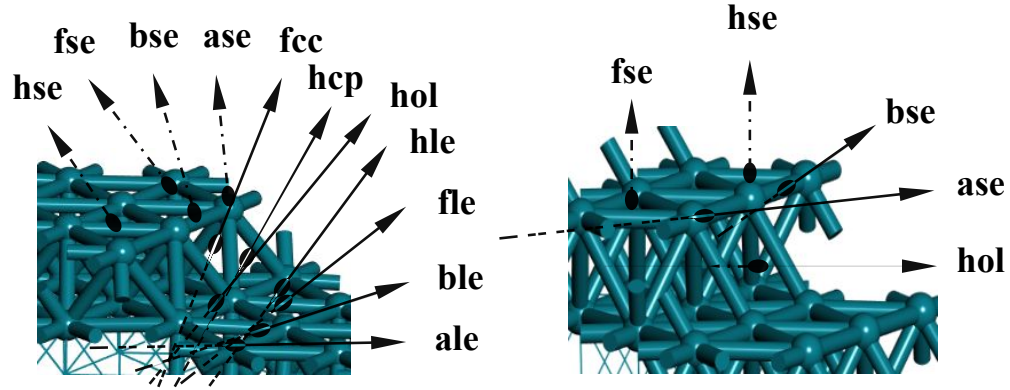

(e) (221)

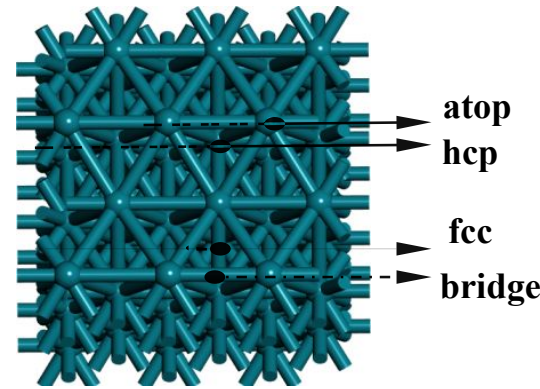

(h) (10-10)

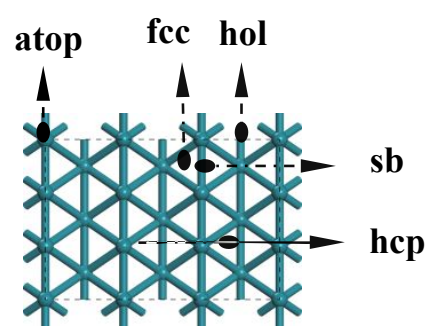

(c) (110) (f) (311)

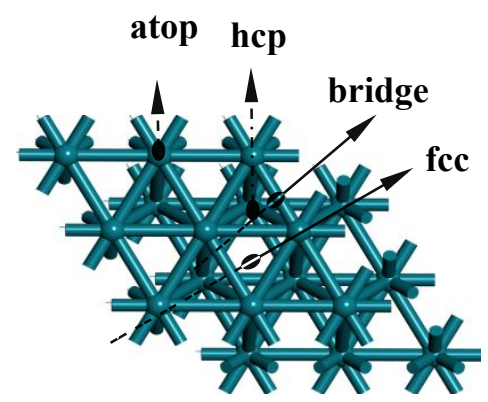

(I) (0001)

Figure S1 The morphologies of different FCC and HCP Rh surfaces and the corresponding adsorption sites. Ase, bse, fse and hse refer to atop, bridge, fcc and hcp sites on the step edge (se). Ale, ble, fle, hle and holle refer to atop, bridge, fcc, hcp and hollow sites on the lower edge (le). Fcc, hcp, and hol represent the 3-fold and 4-fold hollow site, respectively, sb represents the short bridge site. 

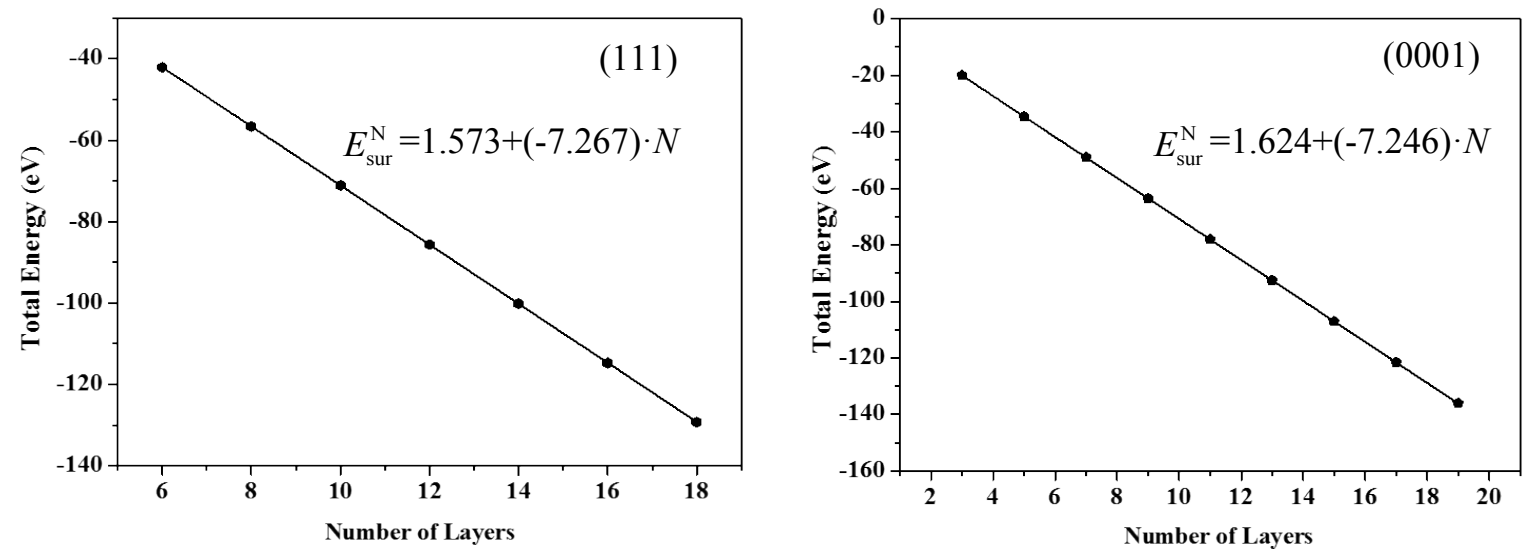

Figure S2 The overall linear fit to the total energy of (111) and (0001) surface slab as a function of the slab layers, respectively.

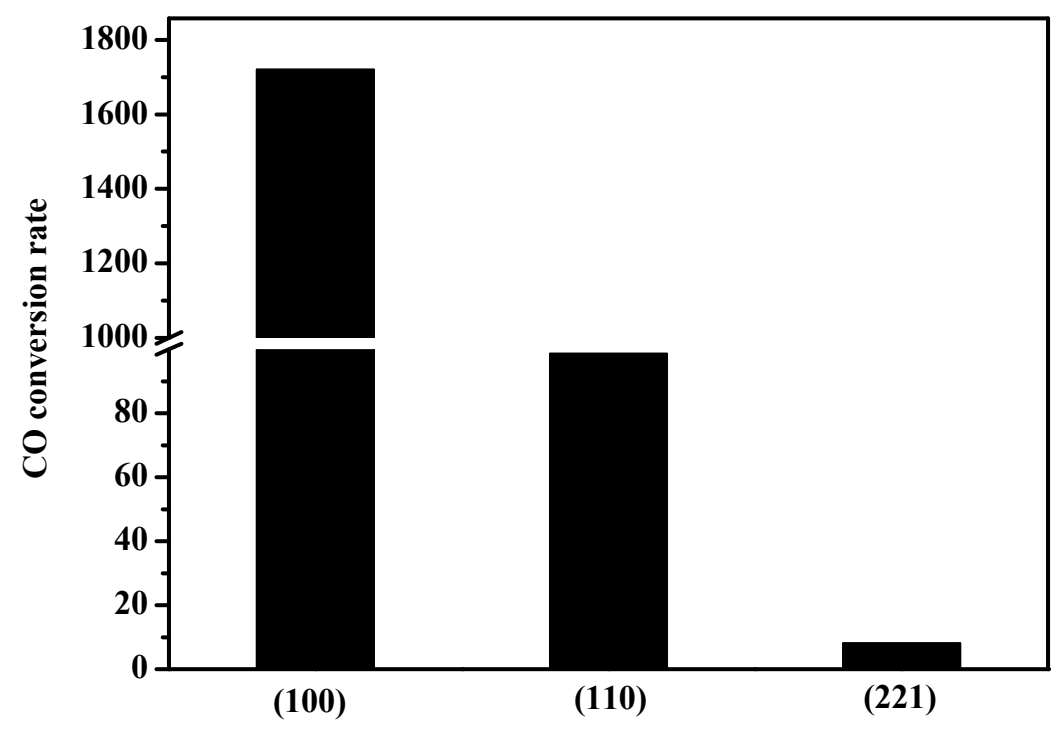

Figure S3 CO conversion rate $\left(\mathrm{s}^{-1}\right)$ on the (100), (100) and (221) surface at the temperature of $543 \mathrm{~K}, \mathrm{H}_{2} / \mathrm{CO}=2$, $\mathrm{H}_{2}$ partial pressure of $8 \mathrm{~atm}$. Assuming FCC Rh is completely covered by (100), (110), or (221). The values are normalized by that of FCC Rh in the text. 
Table S1 The CO adsorption energies $\left(E_{\text {ads }}, \mathrm{eV}\right)$ and the most stable adsorption site on the Rh(111) surface using the different exchange-correlation functional.

\begin{tabular}{ccc}
\hline Functional & $\boldsymbol{E}_{\text {ads }}$ & Site \\
\hline PBE & -1.96 & hcp \\
PBE-D3 & -2.18 & hcp \\
revised PBE & -1.66 & hcp \\
\hline
\end{tabular}


Table S2 The adsorption energies $\left(E_{\mathrm{ads}}, \mathrm{eV}\right)$ and the stable adsorption sites for possible species involving in three CO dissociation pathways on different FCC and HCP Rh surfaces, respectively.

\begin{tabular}{|c|c|c|c|c|c|c|c|c|c|c|c|c|c|c|c|c|}
\hline $\begin{array}{c}\text { FCC } \\
\text { Surfaces }\end{array}$ & $E_{\mathrm{ads}}(\mathrm{CO})$ & Site & $E_{\text {ads }}(C)$ & Site & $E_{\text {ads }}(\mathbf{O})$ & Site & $E_{\text {ads }}(\mathbf{H})$ & Site & $E_{\text {ads }}(\mathrm{CHO})$ & Site & $E_{\text {ads }}(\mathrm{COH})$ & Site & $E_{\text {ads }}(\mathbf{C H})$ & Site & $E_{\text {ads }}(\mathrm{OH})$ & Site \\
\hline$\overline{(111)}$ & -1.96 & hcp & -7.46 & hcp & -5.52 & fcc & -2.78 & fcc & -2.48 & bridge & -4.70 & hcp & -6.90 & hcp & -3.06 & $\overline{\text { bridge }}$ \\
\hline (100) & -1.91 & bridge & -8.22 & hol & -5.40 & hol & -2.73 & hol & -2.84 & hol & -4.77 & hol & -7.10 & hol & -3.33 & bridge \\
\hline (110) & -1.87 & atop & -7.72 & hol & -5.52 & $\mathrm{sb}$ & -2.66 & fcc & -2.67 & hol & -4.20 & fcc & -6.53 & hol & -3.22 & $\mathrm{sb}$ \\
\hline (211) & -1.99 & ase & -7.80 & hol & -5.57 & bse & -2.79 & bse & -2.79 & bse & -4.40 & bse & -6.45 & fse & -3.71 & bse \\
\hline (221) & -1.91 & fse & -7.61 & hol & -5.54 & fse & -2.77 & fse & -2.62 & fse & -4.43 & hse & -6.65 & fse & -3.41 & bse \\
\hline (311) & -2.02 & atop & -7.78 & hol & -5.63 & bse & -2.82 & bridge & -2.84 & bridge & -4.63 & hcp & -6.76 & hol & -3.75 & bridge \\
\hline $\begin{array}{c}\text { HCP } \\
\text { Surfaces }\end{array}$ & $E_{\mathrm{ads}}(\mathrm{CO})$ & Site & $E_{\text {ads }}(C)$ & Site & $E_{\text {ads }}(\mathbf{O})$ & Site & $E_{\text {ads }}(\mathbf{H})$ & Site & $E_{\text {ads }}(\mathrm{CHO})$ & Site & $E_{\text {ads }}(\mathrm{COH})$ & Site & $E_{\text {ads }}(\mathbf{C H})$ & Site & $E_{\text {ads }}(\mathrm{OH})$ & Site \\
\hline$(0001)$ & -2.05 & fcc & -7.78 & hcp & -5.67 & hcp & -2.83 & hcp & -2.64 & hcp & -4.96 & hcp & -7.12 & hcp & -3.22 & hcp \\
\hline$(10-10)$ & -2.07 & bridge & -7.39 & hcp & -5.56 & hcp & -2.81 & hcp & -2.83 & bridge & -4.75 & bridge & -6.90 & $\mathrm{fcc}$ & -3.48 & bridge \\
\hline$(11-20)$ & -2.13 & $\mathrm{sb}$ & -7.43 & hol & -5.47 & $\mathrm{sb}$ & -2.75 & $\mathrm{sb}$ & -2.83 & $\mathrm{sb}$ & -4.47 & fcc & -6.75 & $\mathrm{fcc}$ & -3.49 & $\mathrm{sb}$ \\
\hline$(10-12)$ & -2.05 & fse & -7.77 & holle & -5.81 & bse & -2.83 & fse & -2.83 & bse & -4.59 & fse & -6.85 & fse & -3.59 & bse \\
\hline
\end{tabular}


Table S3 The equilibrium constant $\left(K, \mathrm{~s}^{-1}\right)$, the rate constants $\left(k, \mathrm{~s}^{-1}\right)$ and CO dissociation rate $\left(r_{\text {dis }}\right.$, site $\left.^{-1} \cdot \mathrm{s}^{-1}\right)$ on the exposed surfaces of FCC and HCP Rh crystal phase, respectively.

\begin{tabular}{|c|c|c|c|c|c|c|c|}
\hline Surfaces & CO Dissociation & $K_{1}$ & $K_{2}$ & $\boldsymbol{k}_{3}$ & $k_{-3}$ & $\boldsymbol{k}_{4}$ & $r_{\text {dis }}$ \\
\hline \multicolumn{8}{|c|}{ FCC Rh } \\
\hline$(111)$ & $\mathrm{CO}+\mathrm{H} \rightarrow \mathrm{CHO} \rightarrow \mathrm{CH}+\mathrm{O}$ & $5.67 \times 10^{-12}$ & $1.14 \times 10^{-6}$ & 3.02 & $4.22 \times 10^{9}$ & 1.04 & $4.30 \times 10^{-16}$ \\
\hline$(100)$ & $\mathrm{CO}+\mathrm{H} \rightarrow \mathrm{COH} \rightarrow \mathrm{C}+\mathrm{OH}$ & $1.81 \times 10^{-11}$ & $6.10 \times 10^{-8}$ & $3.78 \times 10^{-3}$ & $1.62 \times 10^{2}$ & 1.22 & $3.10 \times 10^{-11}$ \\
\hline$(311)$ & $\mathrm{CO}+\mathrm{H} \rightarrow \mathrm{COH} \rightarrow \mathrm{C}+\mathrm{OH}$ & $1.41 \times 10^{-12}$ & $3.39 \times 10^{-5}$ & $1.09 \times 10^{-4}$ & $3.31 \times 10^{-7}$ & $2.69 \times 10^{3}$ & $1.03 \times 10^{-21}$ \\
\hline \multicolumn{8}{|c|}{ HCP Rh } \\
\hline$(10-10)$ & $\mathrm{CO}+\mathrm{H} \rightarrow \mathrm{COH} \rightarrow \mathrm{C}+\mathrm{OH}$ & $4.41 \times 10^{-13}$ & $7.52 \times 10^{-9}$ & 0.09 & $2.57 \times 10^{5}$ & 0.01 & $5.51 \times 10^{-17}$ \\
\hline$(0001)$ & $\mathrm{CO}+\mathrm{H} \rightarrow \mathrm{CHO} \rightarrow \mathrm{CH}+\mathrm{O}$ & $3.50 \times 10^{-13}$ & $1.06 \times 10^{-9}$ & 0.99 & $6.18 \times 10^{8}$ & 11.35 & $7.12 \times 10^{-17}$ \\
\hline$(10-12)$ & $\mathrm{CO}+\mathrm{H} \rightarrow \mathrm{CHO} \rightarrow \mathrm{CH}+\mathrm{O}$ & $7.19 \times 10^{-13}$ & $7.19 \times 10^{-5}$ & 53 & $1.56 \times 10^{9}$ & 0.15 & $1.52 \times 10^{-16}$ \\
\hline
\end{tabular}

Table S4 The surface coverages of the intermediates for CO dissociation on the exposed surfaces of FCC and HCP Rh crystal phase, respectively.

\begin{tabular}{ccccc}
\hline Surfaces & $\boldsymbol{\theta}_{\mathbf{C O}}$ & $\boldsymbol{\theta}_{\mathbf{H}}$ & $\boldsymbol{\theta}_{*}$ & $\boldsymbol{\theta}_{\mathbf{C O H}}\left(\boldsymbol{\theta}_{\mathbf{C H O}}\right)$ \\
\hline & & FCC Rh & \\
$(111)$ & $1.17 \times 10^{-6}$ & 0.49 & 0.51 & $8.07 \times 10^{-16}$ \\
$(100)$ & $6.00 \times 10^{-6}$ & 0.18 & 0.82 & $3.10 \times 10^{-11}$ \\
$(311)$ & $9.15 \times 10^{-8}$ & 0.84 & 0.16 & $1.94 \times 10^{-14}$ \\
$(10-10)$ & $1.66 \times 10^{-7}$ & HCP Rh & & \\
$(0001)$ & $1.38 \times 10^{-7}$ & $7.24 \times 10^{-2}$ & 0.93 & $4.77 \times 10^{-15}$ \\
$(10-12)$ & $3.38 \times 10^{-8}$ & $2.85 \times 10^{-2}$ & 0.97 & $6.45 \times 10^{-18}$ \\
\hline
\end{tabular}


Table S5 The number $\left(N_{\text {act }}\right)$ of each activate site, the conversion rate $\left(C, \mathrm{~s}^{-1}\right)$ over the each exposed surface and crystal phase, respectively.

\begin{tabular}{|c|c|c|c|c|}
\hline Crystal Phase & Surfaces & $N_{\text {act }}$ & $C_{\text {sur }}$ & $C_{\text {cry }}$ \\
\hline \multirow{3}{*}{$\mathrm{FCC}$} & (111) & $2.79 \times 10^{19}$ & $7.81 \times 10^{2}$ & \multirow{3}{*}{$3.85 \times 10^{6}$} \\
\hline & (100) & $1.04 \times 10^{18}$ & $3.85 \times 10^{6}$ & \\
\hline & (311) & $1.40 \times 10^{18}$ & $4.06 \times 10^{-5}$ & \\
\hline \multirow{3}{*}{$\mathrm{HCP}$} & $(0001)$ & $3.31 \times 10^{18}$ & 12.88 & \multirow{3}{*}{$1.28 \times 10^{2}$} \\
\hline & $(10-10)$ & $6.55 \times 10^{18}$ & 36.35 & \\
\hline & $(10-12)$ & $6.88 \times 10^{18}$ & 78.43 & \\
\hline
\end{tabular}

Table S6 The change of the activation barrier $\left(\Delta_{\mathrm{a}}\right.$, eV), the adsorption energies of $\mathrm{C}$ and $\mathrm{O}$ atom $\left(\Delta\left(E_{\mathrm{C}}^{\mathrm{TS}}+\right.\right.$ $\left.\left.E_{\mathrm{O}}^{\mathrm{TS}}\right), \mathrm{eV}\right)$ at the TS and the mutual interaction energy $\left(\triangle E_{\mathrm{int}}{ }^{\mathrm{TS}}, \mathrm{eV}\right)$ between $\mathrm{C}$ and $\mathrm{O}$ atom for Class I reaction. Note that the energies given are normalized by those on (211) for comparison.

\begin{tabular}{cccc}
\hline Surfaces & $\Delta \boldsymbol{E}_{\mathrm{a}}$ & $\Delta\left(\boldsymbol{E}_{\mathbf{C}}{ }^{\mathrm{TS}}+\boldsymbol{E}_{\mathbf{O}}{ }^{\mathrm{TS}}\right)$ & $\Delta \boldsymbol{E}_{\mathrm{int}}{ }^{\mathrm{TS}}$ \\
\hline$(111)$ & -0.41 & 0.21 & -0.62 \\
$(100)$ & -2.01 & -2.22 & 0.21 \\
$(110)$ & -1.98 & -1.46 & -0.52 \\
$(221)$ & -0.66 & -0.61 & -0.05 \\
$(311)$ & -0.10 & -0.10 & 0.00 \\
$(0001)$ & -0.60 & -0.11 & -0.49 \\
$(10-10)$ & -0.31 & 0.16 & -0.47 \\
$(11-20)$ & -1.35 & -1.13 & -0.22 \\
$(10-12)$ & -1.48 & -1.93 & 0.45 \\
\hline
\end{tabular}


Table S7 The change of the activation barrier $\left(\Delta E_{\mathrm{a}, 2}, \mathrm{eV}\right)$, the adsorption energies of the intermediate at the IS $\left(\Delta E_{\mathrm{med}}{ }^{\mathrm{IS}}, \mathrm{eV}\right)$, the adsorption energies of $\mathrm{AB}$ and $\mathrm{B}\left(\Delta\left(E_{\mathrm{AB}}^{\mathrm{TS}}+E_{\mathrm{C}}^{\mathrm{TS}}\right), \mathrm{eV}\right)$ at the $\mathrm{TS}$, and the mutual interaction energy $\left(\Delta E_{\mathrm{int}}^{\mathrm{TS}}, \mathrm{eV}\right)$ between $\mathrm{AB}$ and $\mathrm{C}$ for Class II reaction. Note that the energies given are normalized by those on (211) for the reference.

H-assisted CO dissociation via CHO

\begin{tabular}{|c|c|c|c|c|c|c|c|c|}
\hline \multirow{3}{*}{ Surfaces } & $\Delta E_{\mathrm{a}}$ & $\Delta\left(E_{\mathrm{CH}^{\mathrm{TS}}}^{\mathrm{TS}}+E_{\mathrm{O}}{ }^{\mathrm{TS}}\right.$ & $\Delta E_{\mathrm{CHO}}{ }^{\mathrm{I}}$ & \multirow{3}{*}{$\Delta E_{\text {int }}{ }^{\mathrm{TS}}$} & \multirow{3}{*}{$\Delta E_{\mathrm{a}, 2}$} & \multirow[t]{2}{*}{$\Delta\left(E_{\mathrm{C}^{\mathrm{TS}}+E_{\mathrm{OH}}}^{\mathrm{TS}}\right.$} & \multirow[t]{2}{*}{$\Delta E_{\mathrm{COH}}^{\mathrm{I}}$} & \multirow{3}{*}{$\Delta E_{\mathrm{int}}^{\mathrm{TS}}$} \\
\hline & & & & & & & & \\
\hline & 2 & ) & $\mathbf{s}$ & & & ) & $\mathrm{s}$ & \\
\hline (111) & -1.72 & -1.32 & 0.31 & -0.09 & -0.25 & -0.18 & -0.30 & -0.37 \\
\hline (100) & -1.42 & -1.26 & -0.05 & -0.21 & -0.78 & -1.08 & -0.37 & -0.07 \\
\hline (110) & -1.28 & -1.04 & 0.12 & -0.12 & -1.26 & 1.56 & 0.20 & -2.06 \\
\hline (221) & -0.11 & 0.87 & 0.20 & -0.78 & -1.16 & -0.76 & -0.03 & -0.43 \\
\hline (311) & -0.51 & -0.43 & -0.05 & -0.13 & 0.07 & -0.07 & -0.23 & -0.09 \\
\hline$(0001)$ & -1.80 & -1.46 & 0.15 & -0.19 & -0.28 & -0.51 & -0.56 & -0.33 \\
\hline$(10-10)$ & -1.19 & -1.39 & -0.04 & 0.16 & -0.52 & 1.17 & -0.35 & -2.04 \\
\hline$(11-20)$ & -1.10 & -1.26 & -0.04 & 0.12 & -0.52 & -0.58 & -0.07 & -0.01 \\
\hline$(10-12)$ & -1.64 & -1.69 & -0.04 & 0.01 & 0.01 & -0.99 & -0.19 & 0.81 \\
\hline
\end{tabular}

$\mathrm{H}$-assisted $\mathrm{CO}$ dissociation via $\mathrm{COH}$ 


\section{References}

(1) Liu, P.; Rodriguez, J. A.; Water-Gas-Shift Reaction on Metal Nanoparticles and Surfaces. J. Chem. Phys. 2007, $126,164705-1-8$

(2) Liu, P.; Rodriguez, J. A.; Water-Gas-Shift Reaction on Molybdenum Carbide Surfaces: Essential Role of the Oxycarbide. J. Phys. Chem. B 2006, 110, 19418-19425.

(3) http://webbook.nist.gov/chemistry/

(4) Laidler, K. J.; Chemical Kinetics; 3rd ed.; Harper and Row: New York, 1987.

(5) Choi, Y. M.; Liu, P.; Mechanism of Ethanol Synthesis from Syngas on Rh(111). J. Am. Chem. Soc. 2009, 131, 13054-13061.

(6) Fu, X. C.; Shen, W. X.; Yao, T. Y.; Physical chemistry; 4th ed.; Higher Education: Beijing, 1990.

(7) Liu, P.; Logadottir, A.; Nørskov, J. K.; Modeling the Electro-Oxidation of $\mathrm{CO}$ and $\mathrm{H}_{2} / \mathrm{CO}$ on Pt, $\mathrm{Ru}, \mathrm{PtRu}$ and $\mathrm{Pt}_{3}$ Sn. Electrochim. Acta 2003, 48, 3731-3742. 